\title{
Multiobject operational tasks for convex quantum resource theories of state-measurement pairs
}

\author{
Andrés F. Ducuara $\odot, 1,2,3,4,{ }^{*}$ Patryk Lipka-Bartosik $\odot,{ }^{3, \dagger}$ and Paul Skrzypczyk $\odot^{3, \$}$ \\ ${ }^{1}$ Quantum Engineering Centre for Doctoral Training, University of Bristol, Bristol BS8 1FD, United Kingdom \\ ${ }^{2}$ Quantum Engineering Technology Labs, University of Bristol, Bristol BS8 1FD, United Kingdom \\ ${ }^{3}$ H.H. Wills Physics Laboratory, University of Bristol, Tyndall Avenue, Bristol BS8 1TL, United Kingdom \\ ${ }^{4}$ Department of Electrical and Electronic Engineering, University of Bristol, Bristol BS8 1FD, United Kingdom
}

(Received 4 June 2020; accepted 17 August 2020; published 8 September 2020)

\begin{abstract}
The prevalent modus operandi within the framework of quantum resource theories has been to characterize and harness the resources within single objects, in what we can call single-object quantum resource theories. One can wonder, however, whether the resources contained within multiple different types of objects, now in a multiobject quantum resource theory, can simultaneously be exploited for the benefit of an operational task. In this work, we introduce examples of such multiobject operational tasks in the form of subchannel discrimination and subchannel exclusion games, in which the player harnesses the resources contained within the composite object of a state-measurement pair. We prove that for any state-measurement pair in which either of them is resourceful, there exist discrimination and exclusion games for which such a pair outperforms any possible free state-measurement pair. These results hold for arbitrary convex resources of states, and arbitrary convex resources of measurements where the set of free measurements is closed under classical post-processing. Furthermore, we prove that the advantage in these multiobject operational tasks is determined, in a multiplicative manner, by the resource quantifiers of: generalized robustness of resource of both state and measurement for discrimination games and weight of resource of both state and measurement for exclusion games.
\end{abstract}

DOI: 10.1103/PhysRevResearch.2.033374

\section{INTRODUCTION}

The framework of quantum resource theories (QRTs) [1,2] has proven to be a successful approach to quantum information theory. Broadly speaking, it aims at identifying, characterizing, and utilizing quantum phenomena as a resource for fuelling quantum information processing protocols for the development of quantum technologies. A QRT is specified by first defining the objects of the theory, followed by a property of these objects to be regarded as a resource. The choice of a particular property as a resource is then justified by specifying instances, usually in the form of operational tasks, in which the presence of such resourceful object provides an advantage over all resourceless (free) objects. There is a plethora of objects in quantum theory whose properties are deemed as resources, namely, states [3-21], measurements [22-25], behaviours or boxes [26,27], steering assemblages [28], channels [29-33], among many others-see the recent review [2]. Arguably, the most studied QRTs are the ones for states and measurements. QRTs of states address resources such as entanglement [3,4], coherence [5-7], reference frames [8], asymmetry [9], superposition $[10,15]$, purity [11],

\footnotetext{
*andres.ducuara@bristol.ac.uk

†patryk.lipka-bartosik@bristol.ac.uk

†paul.skrzypczyk@bristol.ac.uk
}

Published by the American Physical Society under the terms of the Creative Commons Attribution 4.0 International license. Further distribution of this work must maintain attribution to the author(s) and the published article's title, journal citation, and DOI. athermality [12-14], magic [16], non-Gaussianity [17], and non-Markovianity [18-20]. QRTs of measurements on the other hand, address resources such as nonprojective simulability [22,23], informativeness [24], entanglement, and coherence [25].

Having specified a set of objects and one of their properties to be treated as a resource, it is of interest to quantitatively specify the amount of resource contained within a given object. This can be accomplished by introducing appropriate measures known as resource quantifiers [2]. Two well-known families of these measures are the so-called robustness-based [16,24,34-42] and weight-based [35,43-46] resource quantifiers. These quantifiers have found applications in several scenarios, for instance, at characterizing, the advantage that a resourceful object offers, when compared to all resourceless objects, in specific operational tasks. There are two broad families of such results addressing this quantifier-task correspondence, the so-called robustness-discrimination [24,36,37,4749] and weight-exclusion [50,51] correspondences.

One common feature among all of these results is that they address single-object operational tasks, meaning that a single object is thought of as the resourceful object, and the associated tasks are then exploiting the resource contained within such an individual object. One then can wonder, about the possibility of having operational tasks harnessing two or more different resources out of two, in principle different, objects. We refer to these tasks as multiobject tasks, and we can intuitively approach them from the following two general levels. In a first instance, one can consider a single QRT with two different resources, in which case it is natural to make the distinction of the resources being either: disjoint, intersecting or nested [52]. The case of QRTs of states 
with disjoint resources has been explored in the context of a first law for general QRTs [52], this, inspired by results from the thermodynamics of multiple conserved quantities [53-55]. In a second instance however, one can also consider a multiobject scenario in which a first QRT of certain objects with an arbitrary resource is being specified, followed by a second QRT with different objects with their respective arbitrary resource. We address this latter case by considering a multiobject scenario with two objects, one being a state and a second one being a measurement and therefore, the composite object of interest is now a state-measurement pair.

In this work, we address composite QRTs made of convex QRTs of states with arbitrary resources and convex QRTs of measurements with arbitrary resources where the set of free measurements is closed under classical post-processing (CPP). Taking into account that the set of free measurements is closed under CPP for many important resources for measurements like: entanglement, coherence and informativeness, the results found in this work naturally apply to all of these instances. Explicitly, we introduce multiobject operational tasks in the form of subchannel discrimination and subchannel exclusion games in which, a state-measurement pair is being deemed as the composite object of the theory, as opposed to the state (or the measurement) alone. Interestingly, we find that any resourceful state-measurement pair offers an advantage, over all possible free pairs, when performing at particular multiobject tasks. Furthermore, we find that this advantage can be quantified, in a multiplicative manner, by the amount of resource contained within each object, here measured by the resource quantifiers of generalized robustness and weight, for discrimination and exclusion games respectively. Moreover, these quantifiers also find operational significance in an multiobject encoding-decoding communication task involving the state-measurement pair. We believe that the results found in this work open the door for the exploration of multiobject operational tasks in general convex QRTs with different objects beyond states and measurements.

\section{COMPOSITE CONVEX QUANTUM RESOURCE THEORIES AND MULTIOBJECT OPERATIONAL TASKS}

We start by addressing convex QRTs of states and measurements with arbitrary resources.

Definition 1. (Composite convex QRTs of states and measurements) Consider the set of quantum states in a complex finite-dimensional Hilbert space. A quantum state is an operator $\rho$ satisfying $\rho \geqslant 0$ and $\operatorname{Tr}(\rho)=1$. We consider a property of these states defining a closed convex set which we will call the set of free states and denote as F. We say a state $\rho \in \mathrm{F}$ is a free (resourceless) state and it is resourceful otherwise. We also consider the set of quantum measurements, or positiveoperator valued measures (POVMs) in the same complex finite-dimensional Hilbert space. A POVM is a collection of operators $\mathbb{M}=\left\{M_{a}\right\}, a \in\{1, \ldots, o\}$ with $M_{a} \geqslant 0, \forall a$ and $\sum_{a=1}^{o} M_{a}=\mathbb{1}$. Similarly, we consider a property of measurements defining a closed convex set of free measurements and denote it as $\mathbb{F}$. We say a POVM $\mathbb{M} \in \mathbb{F}$ is a free (resourceless) measurement and it is resourceful otherwise. We say that a state-measurement pair $(\rho, \mathbb{M})$ is: fully free when both state and measurement are free, partially resourceful when either is resourceful, and fully resourceful when both are resourceful.
We now address an operation for measurements.

Definition 2. (Classical post-processing (CPP)) We say that a measurement $\mathbb{N}=\left\{N_{x}\right\}, x \in\{1, \ldots, k\}$ is simulable by the measurement $\mathbb{M}=\left\{M_{a}\right\}, a \in\{1, \ldots, o\}$ when there exists a conditional probability distribution $\{q(x \mid a)\}$ such that $N_{x}=$ $\sum_{a=1}^{o} q(x \mid a) M_{a}, \forall x \in\{1, \ldots, k\}$ [23]. One can check that the simulability of measurements defines a partial order for the set of measurements and therefore we use the notation $\mathbb{N} \preceq \mathbb{M}$, meaning that $\mathbb{N}$ is simulable by $\mathbb{M}$. We refer to this property as simulability of measurements or classical post-processing (CPP).

Intuitively, a set of free measurements is closed under CPP when there is no physical significance to the specific measurement label. For example, when labeling an outcome 0 or 1 does not signify anything. An example where this does not hold is in thermodynamics, where the labels on an energy measurement have physical significance (labelling particular energies) and relabelling is not automatically free, unless the relationship between the label and the energy is also accordingly updated.

We can check that the set of free measurements is closed under CPP for QRTs of measurements with the resources of: entanglement, coherence and informativeness. The sets of free measurements for these resources (separable, coherent, uninformative) are defined by specifying the POVM elements respectively as [24,25]: $M_{x}^{S}=\sum_{k} M_{x, k}^{A} \otimes M_{x, k}^{B}, M_{x}^{C}=$ $\sum_{j} p(x \mid j)|j\rangle\langle j|,\{|j\rangle\}$ a basis of the Hilbert space being considered, and $M_{x}^{U}=p(x) \mathbb{1}$. Since any CPP operation always maps measurements into measurements and can never generate entanglement, coherence nor increase purity, all of these exemplary sets of measurements remain closed under CPP. We will then be addressing, from now on, convex QRTs of measurements with its free set being closed under CPP. We now introduce multiobject operational tasks which are meant to be played with state-measurement pairs.

Definition 3. (Multiobject subchannel discrimination/ exclusion games) Consider a player with access to a statemeasurement pair $(\rho, \mathbb{M})$. The player sends the state $\rho$ to the referee who is in possession of a collection of subchannels $\Psi=\left\{\Psi_{x}\right\}, x \in\{1, \ldots, k\}$. The subchannels $\left\{\Psi_{x}\right\}$ are completely positive (CP) trace-nonincreasing maps, such that $\sum_{x} \Psi_{x}$ forms a completely positive trace-preserving (CPTP) map. The referee promises to apply one of these subchannels on the state $\rho$ and the transformed state is then sent back to the player. The player then effectively has access to the ensemble $\mathcal{E}_{\Psi}^{\rho}=\left\{\rho_{x}, p(x)\right\}$ with $p(x)=\operatorname{Tr}\left[\Psi_{x}(\rho)\right], \rho_{x}=\Psi_{x}(\rho) / p(x)$. In a subchannel discrimination game, the goal is for the player to output a guess $g \in\{1, \ldots, k\}$ for the subchannel that was applied, the player succeeds at the game if $g=x$ and fails when $g \neq x$. In a subchannel exclusion game on the other hand, the goal is for the player to output a guess $g \in\{1, \ldots, k\}$ for a subchannel that was not applied, that is, the player succeeds at the game if $g \neq x$ and fails when $g=x$. In order to generate a guess, the player proceeds to implement the measurement $\mathbb{M}=\left\{M_{a}\right\}$ on the received state and classically post-process the measurement outcome $a$ to produce an output guess $g$, according to a probability distribution $\{p(g \mid a)\}$, for playing either a discrimination or an exclusion game. The probability of success at subchannel discrimination and the 
probability of error at subchannel exclusion are given by

$$
\begin{gathered}
P_{\text {succ }}^{\mathrm{D}}(\Psi, \rho, \mathbb{M})=\max _{\{p(g \mid a)\}} \sum_{x, a, g} \delta_{x, g} p(g \mid a) p(a \mid x) p(x), \\
P_{\mathrm{err}}^{\mathrm{E}}(\Psi, \rho, \mathbb{M})=\min _{\{p(g \mid a)\}} \sum_{x, a, g} \delta_{x, g} p(g \mid a) p(a \mid x) p(x),
\end{gathered}
$$

with $p(a \mid x)=\operatorname{Tr}\left[M_{a} \rho_{x}\right]$ and the maximization (minimization) over all classical post-processing of the measurements outputs $p(g \mid a)$. A subchannel discrimination/exclusion game is specified by the collection of subchannels $\Psi=\left\{\Psi_{x}\right\}$.

A key point to remark, is that the object of interest is now the state-measurement pair $(\rho, \mathbb{M})$, as opposed to the state (or measurement) alone. We now proceed to establish a first result comparing the performance of a fully resourceful statemeasurement pair against all fully free pairs when addressing a particular game.

\section{ANY FULLY RESOURCEFUL STATE-MEASUREMENT PAIR IS USEFUL FOR SUBCHANNEL DISCRIMINATION/EXCLUSION}

Result 1. Consider a convex QRT of states with an arbitrary resource and a convex QRT of measurements with an arbitrary resource closed under CPP. Given a fully resourceful state-measurement pair $(\rho, \mathbb{M})$, meaning that we have both a resourceful state $\rho \notin \mathrm{F}$ and a resourceful measurement $\mathbb{M} \notin$ $\mathbb{F}$, then, there exist subchannel games $\Psi_{D}^{(\rho, \mathbb{M})}$ and $\Psi_{E}^{(\rho, \mathbb{M})}$ such that

$$
\begin{gathered}
\max _{\sigma \in \mathrm{F}} \max _{\mathbb{N} \in \mathbb{F}} P_{\text {succ }}^{\mathrm{D}}\left(\Psi_{D}^{(\rho, \mathbb{M})}, \sigma, \mathbb{N}\right)<P_{\text {succ }}^{\mathrm{D}}\left(\Psi_{D}^{(\rho, \mathbb{M})}, \rho, \mathbb{M}\right), \\
P_{\operatorname{err}}^{\mathrm{E}}\left(\Psi_{E}^{(\rho, \mathbb{M})}, \rho, \mathbb{M}\right)<\min _{\sigma \in \mathrm{F}} \min _{\mathbb{N} \in \mathbb{F}} P_{\mathrm{err}}^{\mathrm{E}}\left(\Psi_{E}^{(\rho, \mathbb{M})}, \sigma, \mathbb{N}\right) .
\end{gathered}
$$

These two strict inequalities mean that the state-measurement pair $(\rho, \mathbb{M})$ provides strictly larger (smaller) advantage (error) than all fully free state-measurement pairs, when playing the subchannel discrimination (exclusion) game specified by $\Psi_{D}^{(\rho, \mathbb{M})}\left(\Psi_{E}^{(\rho, \mathbb{M})}\right)$.

The proof of this result relies on the hyperplane separation theorem [56] as well as on a method first used in the context of quantum steering [36], for "completing" a set of subchannels, from which one can extract suitable operators in order to construct the tailored subchannel games $\Psi_{D}^{(\rho, \mathbb{M})}$ and $\Psi_{E}^{(\rho, \mathbb{M})}$, for which playing with the pair $(\rho, \mathbb{M})$ is optimal. The full proof of this result is in Appendix A. We would now like to quantify this advantage by specifying how large this gap can be. In order to do this, we need to define a suitable resource quantifier for the composite objects of state-measurement pairs. A natural starting point is to quantify the amount of resource contained within the individual objects of interest, states and measurements.

\section{RESOURCE QUANTIFIERS AND MULTIOBJECT GAMES}

We now address resource quantifiers for convex QRTs of states and measurements with arbitrary resources.

Definition 4. (Generalized robustness and weight for states and measurements) Consider a convex QRT of states with an arbitrary resource and a convex QRT of measurements with an arbitrary resource. The generalized robustness and the weight of resource of a state and a measurement are given by

$$
\begin{aligned}
& \mathrm{R}_{\mathrm{F}}(\rho)=\underset{\substack{r \geqslant 0 \\
\sigma \in \mathrm{F} \\
\rho^{G}}}{\min }\left\{r \mid \rho+r \rho^{G}=(1+r) \sigma\right\},
\end{aligned}
$$

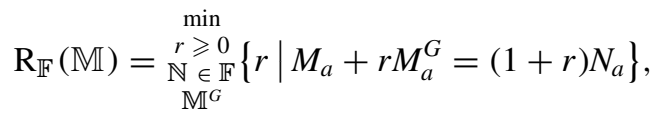

$$
\begin{aligned}
& \mathrm{W}_{\mathrm{F}}(\rho)=\underset{\substack{w \geqslant 0 \\
\sigma \in \mathrm{F} \\
\rho^{G}}}{\min }\left\{w \mid \rho=w \rho^{G}+(1-w) \sigma\right\}, \\
& \mathrm{W}_{\mathbb{F}}(\mathbb{M})=\underset{\substack{w \geqslant 0 \\
\mathbb{N} \in \mathbb{F} \\
\mathbb{M}^{G}}}{\min }\left\{w \mid M_{a}=w M_{a}^{G}+(1-w) N_{a}\right\} .
\end{aligned}
$$

The generalized robustness quantifies the minimum amount of a general state $\rho^{G}$ (measurement $\mathbb{M}^{G}$ ) that has to be added to $\rho(\mathbb{M})$ such that we get a free state $\sigma$ (measurement $\mathbb{N}$ ). The weight on the other hand, quantifies the minimum amount of a general state $\rho^{G}$ (measurement $\mathbb{M}^{G}$ ) that has to be used for recovering the state $\rho$ (measurement $\mathbb{M}$ ).

One now would like to introduce a quantifier for the composite object $(\rho, \mathbb{M})$. It turns out however, that it is enough to quantify the resources contained within the individual objects, as we will see in what follows. We now establish a connection between robustness-based (weight-based) resource quantifiers for states and measurements and multiobject subchannel discrimination (exclusion) games.

Result 2. Consider a convex QRT of states with an arbitrary resource and a convex QRT of measurements with an arbitrary resource closed under CPP. Given any state-measurement pair $(\rho, \mathbb{M})$, we have.

$$
\begin{gathered}
\max _{\Psi} \frac{P_{\text {succ }}^{\mathrm{D}}(\Psi, \rho, \mathbb{M})}{\max _{\substack{\sigma \in \mathrm{F} \\
\mathbb{N} \in \mathbb{F}}} P_{\text {succ }}^{\mathrm{D}}(\Psi, \sigma, \mathbb{N})}=\left[1+\mathrm{R}_{\mathrm{F}}(\rho)\right]\left[1+\mathrm{R}_{\mathbb{F}}(\mathbb{M})\right], \\
\min _{\Psi} \frac{P_{\mathrm{err}}^{\mathrm{E}}(\Psi, \mathbb{M}, \rho)}{\min _{\substack{\sigma \in \mathrm{F} \\
\mathbb{N} \in \mathbb{F}}} P_{\mathrm{err}}^{\mathrm{E}}(\Psi, \sigma, \mathbb{N})}=\left[1-\mathrm{W}_{\mathrm{F}}(\rho)\right]\left[1-\mathrm{W}_{\mathbb{F}}(\mathbb{M})\right],
\end{gathered}
$$

with the maximization (minimization) over all subchannel games.

The full proof of this result is in Appendix B. The first thing we can notice is, that by considering a fully resourceful statemeasurement pair $(\rho, \mathbb{M})$, one recovers the strict inequalities in Eqs. (3) and (4). Additionally, we can also see that by considering now a partially resourceful pair $(\rho, \mathbb{M})$, meaning that either the state or the measurement is resourceful, we still get an advantage. This may seem counter-intuitive at first sight, as using a resourceless measurement should not allow the player to obtain any advantage, even with the most resourceful state. However, as we explicitly show in Appendix B, there still exists a game which allows the player to utilize the advantage arising in such a partially resourceful scenario. The resolution to this apparent paradox is based on the crucial difference between channel and subchannel discrimination/exclusion tasks. In particular, in a subchannel discrimination/exclusion game, a resourceful state has the additional ability to 
"influence" the ensemble of states from which the player needs to discriminate/exclude, since $\mathcal{E}_{\Psi}^{\rho}=\left\{\rho_{x}, p(x)\right\}$ with $p(x)=\operatorname{Tr}\left[\Psi_{x}(\rho)\right], \rho_{x}=\Psi_{x}(\rho) / p(x)$ and therefore, this leads to suitable ensembles, even for resourceless measurements. Similarly, having access to a resourceful measurement provides better guessing strategies, even for ensembles generated by resourceless states. Finally, for a fixed fully free pair, there exists a game for which the pair is still optimal amongst all free pairs. Therefore the ratios considered in result 2 are comparing the performance of any pair against all fully free pairs.

It is illustrative to compare these results with their singleobject counterparts [50,57]. When considering subchannel games being played with a state alone, and allowing maximizations over arbitrary measurements, the advantage becomes $\left[1+\mathrm{R}_{\mathrm{F}}(\rho)\right]$ [57]. In the multiobject scenario considered here however, we get $\left[1+\mathrm{R}_{\mathrm{F}}(\rho)\right]\left[1+\mathrm{R}_{\mathbb{M}}(\mathbb{M})\right]$ instead, which can be larger, whenever $\mathbb{M}$ is resourceful. A similar analysis can be made for the weight-exclusion case [50]. This increment can be conceptually understood by the fact that we are now addressing a composite object and therefore, it is natural that each object contributes to the overall advantage. Nevertheless, it is still surprising that the advantage can be quantified in this elegant multiplicative manner.

It is also interesting to note that this result applies to convex QRTs of states with arbitrary resources and convex QRTs of measurements with arbitrary resources closed under CPP and therefore it covers, as particular instances, several important resources for both states and measurements. It would be interesting to explore whether these results still hold when CPP is dropped or, on the other hand, if a counterexample can be found. We leave this however for future research. We now address multiobject single-shot information-theoretic quantities in the context of an encoding-decoding communication task.

\section{SINGLE-SHOT INFORMATION THEORY}

Consider a state-measurement pair $(\rho, \mathbb{M})$ and the following communication task. Suppose we have an ensemble of subchannels $\Lambda=\left\{\Lambda_{x}\right\}$ which add up to a completely positive and trace preserving map. Our goal is to encode the information about which of these subchannels has been applied in a classical random variable $X$. We do so by applying one of these subchannels to the state $\rho$, resulting in the ensemble of states $\mathcal{E}^{(\Lambda, \rho)}=\left\{\sigma_{x}^{(\Lambda, \rho)}, p(x)\right\}$ with $\sigma_{x}^{(\Lambda, \rho)}=\Lambda_{x}(\rho)$. We refer to the classical random variable $X$ encoded in such a way as $X_{\Lambda, \rho}$. We then consider a decoding scheme using the measurement $\mathbb{M}=\left\{M_{g}\right\}$ with its outcomes representing a (guess) classical random variable $G$. Similarly, we refer to such a decoded variable as $G_{\mathbb{M}}$. We then have that this encoding-decoding scheme depends on the state-measurement pair $(\rho, \mathbb{M})$. A well studied figure of merit for communication tasks is the so-called accessible information [58]. Additionally, it has recently been introduced a complementary figure of merit which has been coined the excludible information, for its natural connection to exclusion tasks [50]. These quantities depend on the plus (minus) infinity mutual information (respectively), which are given by

$$
I_{ \pm \infty}\left(X_{\Lambda, \rho} ; G_{\mathbb{M}}\right)= \pm\left[H_{ \pm \infty}\left(X_{\Lambda, \rho}\right)-H_{ \pm \infty}\left(X_{\Lambda, \rho} \mid G_{\mathbb{M}}\right)\right],
$$

with the order plus and minus infinity entropies $H_{+\infty}\left(X_{\Lambda, \rho}\right)=$ $-\log _{2}\left\{\max _{x} p(x)\right\}$ and $H_{-\infty}\left(X_{\Lambda, \rho}\right)=-\log _{2}\left\{\min _{x} p(x)\right\}$, the order plus and minus infinity conditional entropies $H_{+\infty}\left(X_{\Lambda, \rho} \mid G_{\mathbb{M}}\right)=-\log _{2}\left\{\sum_{g} \max _{x} p(x, g)\right\} \quad$ and $H_{-\infty}\left(X_{\Lambda, \rho} \mid G_{\mathbb{M}}\right)=-\log _{2}\left\{\sum_{g} \min _{x} p(x, g)\right\}$, with $p(x, g)=$ $p(g \mid x) p(x)$ and $p(g \mid x)=\operatorname{Tr}\left[M_{g} \Lambda_{x}(\rho)\right]$. The $\pm \infty$ mutual information quantifies the amount of the respective type of information (accessible or excludible) that can be conveyed by the state-measurement pair and the ensemble of channels at play. These measures are usually functions of the channel but we consider them here as functions of the pair $(\rho, \mathbb{M})$ instead. We now address these quantities for a state-measurement pair in comparison to all fully free pairs.

Result 3. Consider a state-measurement pair $(\rho, \mathbb{M})$. The maximum gap between the plus (minus) infinity mutual information between this pair and all fully free state-measurement pairs is upper bounded as

$$
\begin{aligned}
& \max _{\Lambda}\left\{I_{+\infty}\left(X_{\Lambda, \rho} ; G_{\mathbb{M}}\right)-\max _{\sigma \in \mathrm{F}} \max _{\mathbb{N} \in \mathbb{F}} I_{+\infty}\left(X_{\Lambda, \sigma} ; G_{\mathbb{N}}\right)\right\} \\
& \leqslant \log _{2}\left[1+\mathrm{R}_{\mathrm{F}}(\rho)\right]+\log _{2}\left[1+\mathrm{R}_{\mathbb{F}}(\mathbb{M})\right], \\
& \max _{\Lambda}\left\{I_{-\infty}\left(X_{\Lambda, \rho} ; G_{\mathbb{M}}\right)-\max _{\sigma \in \mathrm{F}} \max _{\mathbb{N} \in \mathbb{F}} I_{-\infty}\left(X_{\Lambda, \sigma} ; G_{\mathbb{N}}\right)\right\} \\
& \quad \leqslant-\log _{2}\left[1-\mathrm{W}_{\mathrm{F}}(\rho)\right]-\log _{2}\left[1-\mathrm{W}_{\mathbb{F}}(\mathbb{M})\right],
\end{aligned}
$$

with the maximization over all ensembles of channels.

The full proof of this result is in Appendix C. This result means that the resource quantifiers place upper bounds for these quantities. It would be interesting to see whether they can be saturated.

\section{CONCLUSIONS}

In this work, we have introduced multiobject operational tasks in which the composite objects of interest are statemeasurement pairs. The results found in this article hold for convex QRTs of states with arbitrary resources and convex QRTs of measurements closed under CPP. In particular, we have shown that any resourceful pair is useful for multiobject subchannel discrimination and exclusion games, when compared to the best possible strategy using fully free statemeasurement pairs.

Furthermore, we have found that this advantage can be quantified, in a multiplicative manner, by the quantifiers of generalized robustness and weight of the state and the measurement, for discrimination and exclusion, respectively. This means that the advantage is always possible whenever at least one of the pair is a resource. This is a consequence of the fact that in our case the resources do not interact with each other (i.e., the set of free objects is the set of all state-measurement pairs in which both of them are free, i.e., the total free set is $\mathrm{F} \times \mathbb{F}$ ). This leads to a natural open question: can we find relevant information-theoretic tasks in situations in which the set of free objects is more complicated, i.e., allows for a nontrivial interplay between the constituent resources? This could be achieved by considering a superset of $\mathrm{F} \times \mathbb{F}$ as the free set and quantifying the quantum advantage in this new case. It would be interesting to see whether this alteration can 
provide any new insights for other information-theoretic tasks and quantifiers.

Moreover, the objects which we jointly studied (state and measurement) are used in subchannel discrimination and exclusion tasks in a way which does not allow them to interfere with each other. It would be interesting to study objects which can influence one another and find tasks exploiting these interactions. For example, one could consider the pair: state and quantum instrument and study the advantage which they provide in tasks with multiple number of guesses. We believe that this may lead to new insights related to the informationdisturbance trade-off purely from a resource theoretic perspective.

Our results also provide support, now in the multiobject regime, to the conjecture made in Ref. [50], about the existence of a weight-exclusion correspondence whenever there is a robustness-discrimination one. We have also introduced a communication task in which the log-robustness and the logweight place upper bounds for information-theoretic quantities.

Finally, we believe that this work opens the door for exploring multiobject operational tasks in general QRTs of arbitrary composite objects with arbitrary resources, beyond those of states and measurements, as well as tasks for pairs of the same type of objects but exploiting different resources, and whether the distinction between the resources being disjoint, intersecting and nested plays any major role.

\section{ACKNOWLEDGMENTS}

We thank an anonymous referee for valuable comments and particularly, for pointing out considering the weaker assumption of the resources being closed under CPP. We also thank Tom Purves for insightful discussions. A.F.D. acknowledges support from COLCIENCIAS 756-2016 and the UK EPSRC (EP/L015730/1). P.L.-B. acknowledges support from the UK EPSRC (grant No. EP/R00644X/1). P.S. acknowledges support from a Royal Society URF (UHQT).

\section{APPENDIX A: PROOF OF RESULT 1}

In order to prove result 1 , we start by rewriting the figures of merit in a more compact form, we then extract some useful operators using the hyperplane separation theorem and define a particular classical post-processing (CPP) operation. With this in place, we proceed to address the discrimination case followed by the exclusion case.

\section{Rewriting the figures of merit}

We start by rewriting the probability of success (error) in multiobject discrimination (exclusion) games in a more compact form. Given a multiobject discrimination game $\Psi=\left\{\Psi_{x}(\cdot)\right\}$ and a state-measurement pair $(\rho, \mathbb{M})$, the probability of success can be written as

$$
\begin{aligned}
& P_{\text {succ }}^{\mathrm{D}}(\Psi, \rho, \mathbb{M}) \\
& =\max _{\{p(g \mid a)\}} \sum_{x, a, g} \delta_{x, g} p(g \mid a) p(a \mid x) p(x), \\
& =\max _{\{p(g \mid a)\}} \sum_{x, a, g} \delta_{x, g} p(g \mid a) \operatorname{Tr}\left[M_{a} \frac{\Psi_{x}(\rho)}{\operatorname{Tr}\left[\Psi_{x}(\rho)\right]}\right] p(x), \\
& =\max _{\{p(g \mid a)\}} \sum_{x, a, g} \delta_{x, g} p(g \mid a) \operatorname{Tr}\left[M_{a} \Psi_{x}(\rho)\right], \\
& =\max _{\{p(g \mid a)\}} \sum_{x} \operatorname{Tr}\left\{\left[\sum_{a}\left(\sum_{g} p(g \mid a) \delta_{x, g}\right) M_{a}\right] \Psi_{x}(\rho)\right\}, \\
& \quad=\max _{\{p(x \mid a)\}} \sum_{x} \operatorname{Tr}\left\{\left[\sum_{a} p(x \mid a) M_{a}\right] \Psi_{x}(\rho)\right\}, \\
& =\max _{\mathbb{N} \preceq \mathbb{M}} \sum_{x} \operatorname{Tr}\left[N_{x} \Psi_{x}(\rho)\right],
\end{aligned}
$$

where in the third line, we used $p(x)=\operatorname{Tr}\left[\Psi_{x}(\rho)\right]$, and in the last line, the maximization is over all measurements simulable by IM. Similarly, for the multiobject exclusion case, we get

$$
P_{\mathrm{err}}^{\mathrm{E}}(\Psi, \rho, \mathbb{M})=\min _{\mathbb{N} \leq \mathbb{M}} \sum_{x} \operatorname{Tr}\left[N_{x} \Psi_{x}(\rho)\right] .
$$

\section{Some useful operators}

Given any fully resourceful state-measurement pair $(\rho, \mathbb{M})$, meaning that $\rho \notin \mathrm{F}$ and $\mathbb{M}=\left\{M_{x}\right\} \notin \mathbb{F}, x \in\{1, \ldots, k\}$ and using the hyperplane separation theorem [56], we have that there exist positive semidefinite operators $Z^{\rho}$ and $\left\{Z_{x}^{\mathbb{M}}\right\}, x \in$ $\{1, \ldots, k\}$ such that

$$
\begin{array}{ll}
\operatorname{Tr}\left(Z^{\rho} \rho\right)>1, & \sum_{x} \operatorname{Tr}\left(Z_{x}^{\mathbb{M}} M_{x}\right)>1, \\
\operatorname{Tr}\left(Z^{\rho} \sigma\right) \leqslant 1, & \sum_{x} \operatorname{Tr}\left(Z_{x}^{\mathbb{M}} N_{x}\right) \leqslant 1,
\end{array}
$$

$\forall \sigma \in \mathrm{F}, \mathbb{N} \in \mathbb{F}$. Similarly, there exist positive semidefinite operators $Y^{\rho}$ and $\left\{Y_{x}^{\mathbb{M}}\right\}, x \in\{1, \ldots, k\}$ such that

$$
\begin{array}{ll}
\operatorname{Tr}\left(Y^{\rho} \rho\right)<1, & \sum_{x} \operatorname{Tr}\left(Y_{x}^{\mathbb{M}} M_{x}\right)<1, \\
\operatorname{Tr}\left(Y^{\rho} \sigma\right) \geqslant 1, & \sum_{x} \operatorname{Tr}\left(Y_{x}^{\mathbb{M}} N_{x}\right) \geqslant 1,
\end{array}
$$

$\forall \sigma \in \mathrm{F}, \mathbb{N} \in \mathbb{F}$. These sets of operators are going to be useful when constructing the subchannel games for discrimination and exclusion.

\section{Particular CPP operation}

Given an arbitrary measurement $\mathbb{N}=\left\{N_{a}\right\}$ with $a \in$ $\{1, \ldots, k+n\}, n$ and $k$ integers, we then construct a measurement $\tilde{\mathbb{N}}=\left\{\tilde{N}_{x}\right\}$ with $k$ elements as

$$
\begin{aligned}
& \tilde{N}_{x}:=N_{x}, \quad x \in\{1, \ldots, k-1\}, \\
& \tilde{N}_{k}:=N_{k}+\sum_{y=k+1}^{k+n} N_{y} .
\end{aligned}
$$


We can check that this is a well-defined measurement and that the operation taking $\mathbb{N}$ into $\tilde{\mathbb{N}}$ is a CPP operation on the initial measurement $\mathbb{N}$. This corresponds to a coarse graining of measurement outcomes, such that any outcome of $\mathbb{N}$ greater or equal than $k$ is declared as outcome $k$.

\section{Discrimination case}

Result 1A. Consider a convex QRT of states with an arbitrary resource and a convex QRT of measurements with an arbitrary resource closed under CPP. Given any fully resourceful state-measurement pair $(\rho, \mathbb{M})$, meaning that we have a resourceful state $\rho \notin \mathrm{F}$ and a resourceful measurement $\mathbb{M} \notin \mathbb{F}$, then, there exists a subchannel game $\Psi^{(\rho, \mathbb{M})}$ such that

$$
\max _{\sigma \in \mathrm{F}} \max _{\mathbb{N} \in \mathbb{F}} P_{\text {succ }}^{\mathrm{D}}\left(\Psi^{(\rho, \mathbb{M})}, \sigma, \mathbb{N}\right)<P_{\text {succ }}^{\mathrm{D}}\left(\Psi^{(\rho, \mathbb{M})}, \rho, \mathbb{M}\right),
$$

with the left side being maximized over all possible free states and free measurements.

Proof. We start by considering a fully resourceful statemeasurement pair $(\rho, \mathbb{M})$. Using the hyperplane separation theorem [56], there exist positive semidefinite operators $Z^{\rho}$ and $\left\{Z_{x}^{\mathbb{M}}\right\}, x \in\{1, \ldots, k\}$ satisfying the conditions (A1) and (A2). We now define the set of maps $\left\{\Phi_{x}^{(\rho, \mathbb{M})}(\cdot)\right\}$ such that for any state $\eta$, we have

$$
\begin{aligned}
\Phi_{x}^{(\rho, \mathbb{M})}(\eta) & :=\alpha^{(\rho, \mathbb{M})} \operatorname{Tr}\left(Z^{\rho} \eta\right) Z_{x}^{\mathbb{M}}, \\
\alpha^{(\rho, \mathbb{M})} & :=\frac{1}{\left\|Z^{\rho}\right\|_{1} \operatorname{Tr}\left(Z^{\mathbb{M}}\right)}, \\
Z^{\mathbb{M}} & :=\sum_{x=1}^{k} Z_{x}^{\mathbb{M}},
\end{aligned}
$$

with $\|X\|_{1}=\operatorname{Tr}\left(\sqrt{X^{\dagger} X}\right)$ the trace norm. We are going to use the notation $\alpha=\alpha^{(\rho, \mathbb{M})}$. We can check that these maps are completely positive and linear, and that they satisfy that $\forall \eta$ :

$$
F(\eta):=\operatorname{Tr}\left[\sum_{x=1}^{k} \Phi_{x}^{(\rho, \mathbb{M})}(\eta)\right]=\frac{\operatorname{Tr}\left(Z^{\rho} \eta\right)}{\left\|Z^{\rho}\right\|_{1}} \leqslant 1 .
$$

The inequality follows from the variational characterization of the trace norm, establishing that $\|X\|_{1}=$ $\max _{-1 \leqslant M \leqslant 1}\{\operatorname{Tr}(X M)\}$ for any Hermitian operator $X$ [58]. We can also write $F(\eta)=\alpha \operatorname{Tr}\left(Z^{\rho} \eta\right) \operatorname{Tr}\left(Z^{\mathbb{M}}\right)$. The set of maps $\left\{\Phi_{x}^{(\rho, \mathbb{M})}(\cdot)\right\}$ then add up to a completely positive tracenonincreasing linear map. We can then complete this set to be a set of subchannels by adding an extra subchannel $\Psi_{k+1}^{(\rho, \mathbb{M})}(\cdot):=\Lambda(\cdot)-\Phi^{(\rho, \mathbb{M})}(\cdot)$, with $\Lambda$ being an arbitrary CPTP map such that it is greater or equal than zero (take the identity channel for instance). Therefore, with this construction, we obtain a well-defined set of subchannels with $k+1$ elements. We now proceed to define a family of sets of subchannels in the following manner. Given a statemeasurement pair $(\rho, \mathbb{M}), \mathbb{M}=\left\{M_{x}\right\}, x \in\{1, \ldots, k\}$, and an integer $n \geqslant 1$, we define the family of sets of subchannels given by $\Psi^{(\rho, \mathbb{M}, n)}=\left\{\Psi_{y}^{(\rho, \mathbb{M}, n)}(\cdot)\right\}, y \in\{1, \ldots, k+n\}$ with:

$$
\Psi_{y}^{(\rho, \mathbb{M}, n)}(\eta):= \begin{cases}\alpha \operatorname{Tr}\left[Z^{\rho} \eta\right] Z_{y}^{\mathbb{M}}, & y=1, \ldots, k \\ \frac{1}{n}[1-F(\eta)] \xi, & y=k+1, \ldots, k+n\end{cases}
$$

with $\xi$ begin an arbitrary quantum state $\xi \geqslant 0, \operatorname{Tr}(\xi)=1$. We can check that this is a well-defined set of subchannels, because they now add up to a CPTP linear map:

$$
\operatorname{Tr}\left[\sum_{y=1}^{k+n} \Psi_{y}^{(\rho, \mathbb{M}, n)}(\eta)\right]=1, \quad \forall n, \forall \eta .
$$

We now analyze the multiobject subchannel discrimination game given by $\Psi^{(\rho, \mathbb{M}, n)}$. The probability of success of a player using the state-measurement pair $(\rho, \mathbb{M})$ is given by

$$
\begin{aligned}
P_{\text {succ }}^{\mathrm{D}}\left(\Psi^{(\rho, \mathbb{M}, n)} \rho, \mathbb{M}\right) & =\max _{\mathbb{N} \leq \mathbb{M}} \sum_{y=1}^{k+n} \operatorname{Tr}\left[N_{y} \Psi_{y}^{(\rho, \mathbb{M}, n)}(\rho)\right] \\
& \geqslant \sum_{x=1}^{k} \operatorname{Tr}\left[M_{x} \Psi_{x}^{(\rho, \mathbb{M}, n)}(\rho)\right] \\
& =\alpha \operatorname{Tr}\left[Z^{\rho} \rho\right] \sum_{x=1}^{k} \operatorname{Tr}\left[M_{x} Z_{x}^{\mathbb{M}}\right] .
\end{aligned}
$$

The inequality follows because we have chosen to simulate a particular measurement, i.e. $N_{y}=M_{y}$ for $y \leqslant k$ and $N_{y}=0$ for $y>k$. In the last equality, we have replaced the subchannel discrimination game with (A7). Now, because of the conditions in Eq. (A1), we have the strict inequality:

$$
P_{\text {succ }}^{\mathrm{D}}\left(\Psi^{(\rho, \mathbb{M}, n)}, \rho, \mathbb{M}\right)>\alpha .
$$

We now analyze the best fully free player:

$$
\max _{\substack{\sigma \in \mathrm{F} \\ \mathbb{N} \in \mathbb{F}}} P_{\text {succ }}^{\mathrm{D}}\left(\Psi^{(\rho, \mathbb{M}, n)}, \sigma, \mathbb{N}\right)=\max _{\substack{\sigma \in \mathrm{F} \\ \mathbb{N} \in \mathbb{F} \\ \tilde{\mathbb{N}} \preceq \mathbb{N}}} \sum_{x=1}^{k+n} \operatorname{Tr}\left[\tilde{N}_{x} \Psi_{x}^{(\rho, \mathbb{M}, n)}(\sigma)\right] .
$$

We are considering QRTs of measurements closed under CPP and therefore, CPP is redundant here and we have

$$
\max _{\substack{\sigma \in \mathrm{F} \\ \mathbb{N} \in \mathbb{F}}} P_{\text {succ }}^{\mathrm{D}}\left(\Psi^{(\rho, \mathbb{M}, n)}, \sigma, \mathbb{N}\right)=\max _{\substack{\sigma \in \mathrm{F} \\ \mathbb{N} \in \mathbb{F}}} \sum_{x=1}^{k+n} \operatorname{Tr}\left[N_{x} \Psi_{x}^{(\rho, \mathbb{M}, n)}(\sigma)\right] .
$$

Let us now consider, without loss of generality, that these two maximizations are being achieved by the fully free pair $\left(\sigma^{*}, \mathbb{N}^{*}\right)$. We then have

$$
\begin{aligned}
P_{\text {succ }}^{\mathrm{D}}\left(\Psi^{(\rho, \mathbb{M}, n)}, \sigma^{*}, \mathbb{N}^{*}\right)= & \sum_{x=1}^{k+n} \operatorname{Tr}\left[N_{x}^{*} \Psi_{x}^{(\rho, \mathbb{N}, n)}\left(\sigma^{*}\right)\right], \\
= & \alpha \operatorname{Tr}\left[Z^{\rho} \sigma^{*}\right] \sum_{y=1}^{k} \operatorname{Tr}\left[N_{y}^{*} Z_{y}^{\mathbb{M}}\right] \\
& +\frac{1}{n}\left[1-F\left(\sigma^{*}\right)\right] \sum_{y=k+1}^{k+n} \operatorname{Tr}\left[N_{y}^{*} \xi\right] .
\end{aligned}
$$

In the second equality, we have replaced the subchannel game (A7). The first term can be upper bounded as

$$
\sum_{y=1}^{k} \operatorname{Tr}\left[N_{y}^{*} Z_{y}^{\mathbb{M}}\right] \leqslant \sum_{y=1}^{k} \operatorname{Tr}\left[\tilde{N}_{y}^{*} Z_{y}^{\mathbb{M}}\right] \leqslant 1,
$$


with the measurement $\tilde{\mathbb{N}}^{*}$ defined in Eq. (A5). The first inequality follows from the definition of the measurement $\tilde{\mathbb{N}}^{*}$. In the second inequality we use the fact that $\tilde{\mathbb{N}}^{*}$ is a free measurement (because it was constructed from a free measurement $\mathbb{N}^{*}$ and a CPP operation) and therefore we can use the conditions in Eq. (A2). We now also use the fact that $1-F(\eta) \leqslant 1, \forall \eta$, then equation (A10) becomes

$$
P_{\text {succ }}^{\mathrm{D}}\left(\Psi^{(\rho, \mathbb{M}, n)}, \sigma, \mathbb{N}\right) \leqslant \alpha+\frac{1}{n} \sum_{y=k+1}^{k+n} \operatorname{Tr}\left[N_{y}^{*} \xi\right] .
$$

The second term can be upper bounded as

$$
\sum_{y=k+1}^{k+n} \operatorname{Tr}\left[N_{y}^{*} \xi\right] \leqslant \sum_{y=1}^{k+n} \operatorname{Tr}\left[N_{y}^{*} \xi\right]=\operatorname{Tr}\left[\left(\sum_{y=1}^{k+n} N_{y}^{*}\right) \xi\right]=1 .
$$

The inequality follows because we have added positive terms and the equality follows from $\mathbb{N}^{*}$ being a measurement $\sum_{y=1}^{k+n} \tilde{N}_{y}=\mathbb{1}$ and $\xi$ being a state. We then get

$$
P_{\text {succ }}^{\mathrm{D}}\left(\Psi^{(\rho, \mathbb{M}, n)}, \sigma, \mathbb{N}\right) \leqslant \alpha+\frac{1}{n} .
$$

We now choose the subchannel game given by $\Psi^{(\rho, \mathbb{M}, n \rightarrow \infty)}$ and therefore we get

$$
P_{\text {succ }}^{\mathrm{D}}\left(\Psi^{(\rho, \mathbb{M}, n \rightarrow \infty)}, \sigma, \mathbb{N}\right) \leqslant \alpha .
$$

Finally, Eqs. (A9) and (A11) together imply that

$$
\max _{\substack{\sigma \in \mathrm{F} \\ \mathbb{N} \in \mathbb{F}}} P_{\text {succ }}^{\mathrm{D}}\left(\Psi^{(\rho, \mathbb{M}, n \rightarrow \infty)}, \sigma, \mathbb{N}\right)<P_{\text {succ }}^{\mathrm{D}}\left(\Psi^{(\rho, \mathbb{M}, n \rightarrow \infty)}, \rho, \mathbb{M}\right),
$$

as desired.

\section{Exclusion case}

Result 1B. Consider a convex QRT of states with an arbitrary resource and a convex QRT of measurements with an arbitrary resource closed under CPP. Given any fully resourceful state-measurement pair, meaning that we have a resourceful state $\rho \notin \mathrm{F}$ and a resourceful measurement $\mathbb{M} \notin \mathbb{F}$, then, there exist a subchannel game $\Psi^{(\rho, \mathbb{M})}$ such that

$$
P_{\text {err }}^{\mathrm{E}}\left(\Psi^{(\rho, \mathbb{M})}, \rho, \mathbb{M}\right)<\min _{\sigma \in \mathrm{F}} \min _{\mathbb{N} \in \mathbb{F}} P_{\text {err }}^{\mathrm{E}}\left(\Psi^{(\rho, \mathbb{M})}, \sigma, \mathbb{N}\right),
$$

with minimization over all possible free states and measurements.

Proof. This proof is closely related to the discrimination proof, but the subchannel game has to be constructed differently. We start by considering a fully resourceful statemeasurement pair $(\rho, \mathbb{M})$. Using the hyperplane separation theorem [56], there exist positive semidefinite operators $Y^{\rho}$ and $\left\{Y_{x}^{\mathbb{M}}\right\}, x \in\{1, \ldots, k\}$ satisfying the conditions (A3) and (A4). We now define the set of maps $\left\{\Phi_{x}^{(\rho, \mathbb{M})}(\cdot)\right\}$ with

$$
\begin{aligned}
\Phi_{x}^{(\rho, \mathbb{M})}(\eta) & :=\beta^{(\rho, \mathbb{M})} \operatorname{Tr}\left(Y^{\rho} \eta\right) Y_{x}^{\mathbb{M}}, \\
\beta^{(\rho, \mathbb{M})} & :=\frac{1}{2\left\|Y^{\rho}\right\|_{1} \operatorname{Tr}\left(Y^{\mathbb{M}}\right)}, \\
Y^{\mathbb{M}} & :=\sum_{x=1}^{k} Y_{x}^{\mathbb{M}},
\end{aligned}
$$

with $\|X\|_{1}=\operatorname{Tr}\left(\sqrt{X^{\dagger} X}\right)$ the trace norm. We are going to use the notation $\beta=\beta^{(\rho, \mathbb{M})}$. As before, these operators are completely positive linear maps and they now satisfy that $\forall \eta$ :

$$
G(\eta):=\operatorname{Tr}\left[\sum_{x=1}^{k} \Phi_{x}^{(\rho, \mathbb{M})}(\eta)\right]=\frac{\operatorname{Tr}\left(Y^{\rho} \eta\right)}{2\left\|Y^{\rho}\right\|_{1}} \leqslant \frac{1}{2},
$$

which can also be written as

$$
G(\eta)=\beta \operatorname{Tr}\left(Y^{\rho} \eta\right) \operatorname{Tr}\left(Y^{\mathbb{M}}\right) .
$$

The set of maps $\left\{\Phi_{x}^{(\rho, \mathbb{M})}(\cdot)\right\}$ then add up to a completely positive trace-nonincreasing linear map. We can then complete this set to be a set of subchannels by adding an extra subchannel $\Psi_{k+1}^{(\rho, \mathbb{M})}(\cdot):=\Lambda(\cdot)-\Phi^{(\rho, \mathbb{M})}(\cdot)$, with $\Lambda$ being an arbitrary CPTP map such that it is greater or equal than zero (take the identity channel for instance). Therefore, with this construction we obtain a well-defined set of subchannels with $k+1$ elements. We now proceed to define a set of subchannels in the following manner. Given a state-measurement pair $(\rho, \mathbb{M}), \mathbb{M}=\left\{M_{x}\right\}, x \in\{1, \ldots, k\}$, we define the set of subchannels given by $\Psi^{(\rho, \mathbb{M})}=\left\{\Psi_{y}^{(\rho, \mathbb{M})}(\cdot)\right\}, y \in\{1, \ldots, k+n\}$ with:

$$
\Psi_{y}^{(\rho, \mathbb{M})}(\eta):=\left\{\begin{array}{lc}
\beta \operatorname{Tr}\left[Y^{\rho} \eta\right] Y_{y}^{\mathbb{M}}, & y=1, \ldots, k \\
{[1-G(\eta)] \xi^{\mathbb{M}},} & y=k+1
\end{array}\right.
$$

with the quantum state

$$
\xi^{\mathbb{M}}:=\frac{\sum_{x=1}^{k} p(x) Y_{x}^{\mathbb{M}}}{\sum_{x=1}^{k} p(x) \operatorname{Tr}\left(Y_{x}^{\mathbb{M}}\right)} .
$$

$\{p(x)\}$ being an arbitrary probability distribution. We can also check that this is a well-defined set of subchannels, i.e., they add up to a CPTP linear map:

$$
\operatorname{Tr}\left[\sum_{y=1}^{k+1} \Psi_{y}^{(\rho, \mathbb{M})}(\eta)\right]=1, \quad \forall \eta .
$$

We remark here that, unlike the discrimination case, we are not generating a family of sets of subchannels, but only a specific one. We now analyze the multiobject subchannel exclusion game given by $\Psi^{(\rho, \mathbb{M})}$ and the probability of error of a player using the state-measurement pair $(\rho, \mathbb{M})$, which is given by

$$
\begin{aligned}
P_{\mathrm{err}}^{\mathrm{E}}\left(\Psi^{(\rho, \mathbb{M})} \rho, \mathbb{M}\right) & =\min _{\mathbb{N} \leq \mathbb{M}} \sum_{y=1}^{k+n} \operatorname{Tr}\left[N_{y} \Psi_{y}^{(\rho, \mathbb{M})}(\rho)\right] \\
& \leqslant \sum_{x=1}^{k} \operatorname{Tr}\left[M_{x} \Psi_{x}^{(\rho, \mathbb{M})}(\rho)\right] \\
& =\beta \operatorname{Tr}\left[Y^{\rho} \rho\right] \sum_{x=1}^{k} \operatorname{Tr}\left[M_{x} Y_{x}^{\mathbb{M}}\right] .
\end{aligned}
$$

The inequality follows because we have chosen to simulate a particular measurement, i.e. $N_{y}=M_{y}$ for $y \leqslant k$ and $N_{y}=0$ for $y>k$. In the last equality, we have replaced the subchannel exclusion game with (A15). Now, because of (A3) and (A4), we have the strict inequality:

$$
P_{\mathrm{err}}^{\mathrm{E}}\left(\Psi^{(\rho, \mathbb{M})}, \rho, \mathbb{M}\right)<\beta .
$$


As before, we now analyze the best fully free player:

$$
\begin{aligned}
\min _{\substack{\sigma \in \mathrm{F} \\
\mathbb{N} \in \mathbb{F}}} P_{\mathrm{err}}^{\mathrm{E}}\left(\Psi^{(\rho, \mathbb{M})}, \sigma, \mathbb{N}\right)=\min _{\substack{\sigma \in \mathrm{F} \\
\mathbb{N} \in \mathbb{F} \\
\mathbb{N} \preceq \mathbb{N}}} \sum_{x=1}^{k+1} \operatorname{Tr}\left[\tilde{N}_{x} \Psi_{x}^{(\rho, \mathbb{M})}(\sigma)\right] \\
=\min _{\substack{\sigma \in \mathrm{F} \\
\mathbb{N} \in \mathbb{F}}} \sum_{x=1}^{k+1} \operatorname{Tr}\left[N_{x} \Psi_{x}^{(\rho, \mathbb{M})}(\sigma)\right],
\end{aligned}
$$

where the equality follows because CPP is redundant. Let us now consider, without loss of generality, that these two minimizations are achieved by the fully free pair $\left(\sigma^{*}, \mathbb{N}^{*}\right)$. We then have

$$
\begin{aligned}
P_{\mathrm{err}}^{\mathrm{E}}\left(\Psi^{(\rho, \mathbb{M})}, \sigma^{*}, \mathbb{N}^{*}\right)= & \sum_{x=1}^{k+1} \operatorname{Tr}\left[N_{x}^{*} \Psi_{x}^{(\rho, \mathbb{N})}\left(\sigma^{*}\right)\right] \\
= & \beta \operatorname{Tr}\left[Y^{\rho} \sigma\right] \sum_{y=1}^{k} \operatorname{Tr}\left[N_{y}^{*} Y_{y}^{\mathbb{M}}\right] \\
& +\left[1-G\left(\sigma^{*}\right)\right] \operatorname{Tr}\left[N_{k+1}^{*} \xi^{\mathbb{M}}\right] .
\end{aligned}
$$

We now add and subtract a convenient term as

$$
\begin{aligned}
P_{\mathrm{err}}^{\mathrm{E}}\left(\Psi^{(\rho, \mathbb{M})}, \sigma^{*}, \mathbb{N}^{*}\right)= & \beta \operatorname{Tr}\left[Y^{\rho} \sigma\right] \sum_{x=1}^{k} \operatorname{Tr}\left[N_{x}^{*} Y_{x}^{\mathbb{M}}\right] \\
& +\beta \operatorname{Tr}\left(Y^{\rho} \sigma^{*}\right) \sum_{x=1}^{k} p(x) \operatorname{Tr}\left(N_{k+1}^{*} Y_{x}^{\mathbb{M}}\right) \\
& -\beta \operatorname{Tr}\left(Y^{\rho} \sigma^{*}\right) \sum_{x=1}^{k} p(x) \operatorname{Tr}\left(N_{k+1}^{*} Y_{x}^{\mathbb{M}}\right) \\
& +\left[1-G\left(\sigma^{*}\right)\right] \operatorname{Tr}\left[N_{k+1}^{*} \xi^{\mathbb{M}}\right] .
\end{aligned}
$$

We now define a measurement given by $\tilde{\mathbb{N}}=\left\{\tilde{N}_{x}^{*}\right\}$ with $\tilde{N}_{x}^{*}=$ $N_{x}^{*}+p(x) N_{k+1}^{*}$, and $p(x)$ being the probability distribution from (A16), and we can reorganize this as

$$
\begin{aligned}
P_{\mathrm{err}}^{\mathrm{E}}\left(\Psi^{(\rho, \mathbb{M})}, \sigma^{*}, \mathbb{N}^{*}\right)= & \beta \operatorname{Tr}\left[Y^{\rho} \sigma\right] \sum_{y=1}^{k} \operatorname{Tr}\left[\tilde{N}_{y}^{*} Y_{y}^{\mathbb{M}}\right] \\
& +\left[1-G\left(\sigma^{*}\right)\right] \operatorname{Tr}\left[N_{k+1}^{*} \xi^{\mathbb{M}}\right] \\
& -\beta \operatorname{Tr}\left(Y^{\rho} \sigma^{*}\right) \sum_{x=1}^{k} p(x) \operatorname{Tr}\left(N_{k+1}^{*} Y_{x}^{\mathbb{M}}\right) .
\end{aligned}
$$

The first term is lower bounded by $\beta$ by using the conditions in Eq. (A4) and therefore we have

$$
\begin{aligned}
P_{\mathrm{err}}^{\mathrm{E}} & \left(\Psi^{(\rho, \mathbb{M}, n)}, \sigma^{*}, \mathbb{N}^{*}\right) \\
\geqslant & \beta+\left[1-G\left(\sigma^{*}\right)\right] \operatorname{Tr}\left[N_{k+1}^{*} \xi^{\mathbb{M}}\right] \\
& \quad-\beta \operatorname{Tr}\left(Y^{\rho} \sigma^{*}\right) \sum_{x=1}^{k} p(x) \operatorname{Tr}\left(N_{k+1}^{*} Y_{x}^{\mathbb{M}}\right) .
\end{aligned}
$$

We now prove that the remaining term (last two lines) is always greater than or equal to zero. We start by rewriting this term as

$$
\begin{aligned}
& {\left[1-G\left(\sigma^{*}\right)\right] \operatorname{Tr}\left[N_{k+1}^{*} \xi^{\mathbb{M}}\right]} \\
& -\beta \operatorname{Tr}\left(Y^{\rho} \sigma^{*}\right) \sum_{x=1}^{k} p(x) \operatorname{Tr}\left(N_{k+1}^{*} Y_{x}^{\mathbb{M}}\right) \\
& =\operatorname{Tr}\left\{N _ { k + 1 } ^ { * } \left[\left(1-G\left(\sigma^{*}\right)\right) \xi^{\mathbb{M}}\right.\right. \\
& \left.\left.\quad-\beta \operatorname{Tr}\left(Y^{\rho} \sigma^{*}\right) \sum_{x=1}^{k} p(x) Y_{x}^{\mathbb{M}}\right]\right\} .
\end{aligned}
$$

We have $N_{k+1}^{*} \geqslant 0$ and therefore we now only need to prove that the operator inside the square brackets is positive semidefinite. We rewrite this operator as

$$
\begin{gathered}
{\left[1-G\left(\sigma^{*}\right)\right] \xi^{\mathbb{M}}-\beta \operatorname{Tr}\left(Y^{\rho} \sigma^{*}\right) \sum_{x=1}^{k} p(x) Y_{x}^{\mathbb{M}}} \\
=\left[1-G\left(\sigma^{*}\right)\right] \frac{\sum_{x=1}^{k} p(x) Y_{x}^{\mathbb{M}}}{\sum_{x=1}^{k} p(x) \operatorname{Tr}\left(Y_{x}^{\mathbb{M}}\right)} \\
\quad-\beta \operatorname{Tr}\left(Y^{\rho} \sigma^{*}\right) \sum_{x=1}^{k} p(x) Y_{x}^{\mathbb{M}}
\end{gathered}
$$

where we used (A16) to substitute for $\xi^{\mathbb{M}}$. We now multiply by the positive term $\sum_{x=1}^{k} p(x) \operatorname{Tr}\left(Y_{x}^{\mathbb{M}}\right)$ and obtain

$$
\begin{aligned}
& {\left[1-G\left(\sigma^{*}\right)\right] \sum_{x=1}^{k} p(x) Y_{x}^{\mathbb{M}}} \\
& \quad-\beta \operatorname{Tr}\left(Y^{\rho} \sigma^{*}\right)\left(\sum_{x=1}^{k} p(x) \operatorname{Tr}\left(Y_{x}^{\mathbb{M}}\right)\right)\left(\sum_{x=1}^{k} p(x) Y_{x}^{\mathbb{M}}\right) .
\end{aligned}
$$

We now factorize the positive semidefinite operator $\sum_{x=1}^{k} p(x) Y_{x}^{\mathbb{M}}$ and analyze the coefficient as follows:

$$
\begin{aligned}
1- & G\left(\sigma^{*}\right)-\beta \operatorname{Tr}\left(Y^{\rho} \sigma^{*}\right) \sum_{x=1}^{k} p(x) \operatorname{Tr}\left(Y_{x}^{\mathbb{M}}\right) \\
& =1-\beta \operatorname{Tr}\left(Y^{\rho} \sigma^{*}\right) \operatorname{Tr}\left(Y^{\mathbb{M}}\right)-\beta \operatorname{Tr}\left(Y^{\rho} \sigma^{*}\right) \sum_{x=1}^{k} p(x) \operatorname{Tr}\left(Y_{x}^{\mathbb{M}}\right), \\
& \geqslant 1-2 \beta \operatorname{Tr}\left(Y^{\rho} \sigma^{*}\right) \operatorname{Tr}\left(Y^{\mathbb{M}}\right) \\
& =1-\frac{\operatorname{Tr}\left(Y^{\rho} \sigma^{*}\right)}{\left\|Y^{\rho}\right\|_{1}} \geqslant 0 .
\end{aligned}
$$

In the first equality, we replaced $G\left(\sigma^{*}\right)$ using (A14). The first inequality follows because we are subtracting a larger quantity. In the second equality we substituted $\beta$ (A13). The second inequality follows because $\frac{\operatorname{Tr}\left(Y^{\rho} \eta\right)}{\left\|Y^{\rho}\right\|_{1}} \leqslant 1, \forall \eta$. Coming back to (A19) we then have

$$
P_{\mathrm{err}}^{\mathrm{E}}\left(\Psi^{(\rho, \mathbb{M})}, \sigma^{*}, \mathbb{N}^{*}\right) \geqslant \beta .
$$

Putting together (A18) and (A21), we obtain

$$
P_{\mathrm{err}}^{\mathrm{E}}\left(\Psi^{(\rho, \mathbb{M})}, \rho, \mathbb{M}\right)<\min _{\sigma \in \mathrm{F}} \min _{\mathbb{N} \in \mathbb{F}} P_{\mathrm{err}}^{\mathrm{E}}\left(\Psi^{(\rho, \mathbb{M})}, \sigma, \mathbb{N}\right),
$$

as desired. 


\section{APPENDIX B: PROOF OF RESULT 2}

We divide this result in two parts. In the first part, we prove the upper bound for discrimination and the lower bound for exclusion. In the second part, we show how to achieve these bounds.

Result 2A. Consider a convex QRT of states with an arbitrary resource and a convex QRT of measurements with an arbitrary resource closed under CPP. Given any statemeasurement pair $(\rho, \mathbb{M})$, we have

$$
\max _{\Psi} \frac{P_{\text {succ }}^{\mathrm{D}}(\Psi, \rho, \mathbb{M})}{\max _{\substack{\sigma \in \mathrm{F} \\ \mathbb{N} \in \mathbb{F}}} P_{\text {succ }}^{\mathrm{D}}(\Psi, \sigma, \mathbb{N})}=\left[1+\mathrm{R}_{\mathrm{F}}(\rho)\right]\left[1+\mathrm{R}_{\mathbb{F}}(\mathbb{M})\right] .
$$

Result 2B. Consider a convex QRT of states with an arbitrary resource and a convex QRT of measurements with an arbitrary resource closed under CPP. Given any statemeasurement pair $(\rho, \mathbb{M})$, we have

$$
\min _{\Psi} \frac{P_{\mathrm{err}}^{\mathrm{E}}(\Psi, \rho, \mathbb{M})}{\min _{\substack{\sigma \in \mathrm{F} \\ \mathbb{N} \in \mathbb{F}}} P_{\mathrm{err}}^{\mathrm{E}}(\Psi, \sigma, \mathbb{N})}=\left[1-\mathrm{W}_{\mathrm{F}}(\rho)\right]\left[1-\mathrm{W}_{\mathbb{F}}(\mathbb{M})\right]
$$

\section{Upper bound for multiobject discrimination and lower bound for multiobject exclusion}

We start by proving that for any state-measurement pair $(\rho, \mathbb{M})$, the product $\left[1+\mathbf{R}_{\mathrm{F}}(\rho)\right]\left[1+\mathbf{R}_{\mathbb{F}}(\mathbb{M})\right]$ places an upper bound on the advantage ratio in any subchannel game $\Psi$.

Proof. Given any subchannel game $\Psi$ and any statemeasurement pair $(\rho, \mathbb{M})$, we have

$$
\begin{aligned}
P_{\text {succ }}^{\mathrm{D}}(\Psi, \rho, \mathbb{M})=\max _{\mathbb{N} \leq \mathbb{M}} \sum_{x} \operatorname{Tr}\left[N_{x} \Psi_{x}(\rho)\right] & \leqslant\left[1+\mathrm{R}_{\mathrm{F}}(\rho)\right] \max _{\mathbb{N} \leq \mathbb{M}} \sum_{x} \operatorname{Tr}\left[N_{x} \Psi_{x}\left(\sigma^{*}\right)\right], \\
& \leqslant\left[1+\mathrm{R}_{\mathrm{F}}(\rho)\right] \max _{\sigma \in \mathrm{F}} \max _{\mathbb{N} \leq \mathbb{M}} \sum_{x} \operatorname{Tr}\left[N_{x} \Psi_{x}(\sigma)\right], \\
& =\left[1+\mathrm{R}_{\mathrm{F}}(\rho)\right] \max _{\sigma \in \mathrm{F}} \max _{\{q(x \mid a)\}} \sum_{x} \operatorname{Tr}\left[\left(\sum_{a} q(x \mid a) M_{a}\right) \Psi_{x}(\sigma)\right], \\
& \leqslant\left[1+\mathrm{R}_{\mathrm{F}}(\rho)\right]\left[1+\mathrm{R}_{\mathbb{F}}(\mathbb{M})\right] \max _{\sigma \in \mathrm{F}} \max _{\{q(x \mid a)\}} \sum_{x} \operatorname{Tr}\left[\left(\sum_{a} q(x \mid a) \tilde{N}_{a}^{*}\right) \Psi_{x}(\sigma)\right], \\
& \left.=\left[1+\mathrm{R}_{\mathrm{F}}(\rho)\right]\left[1+\mathrm{R}_{\mathbb{F}}(\mathbb{M})\right] \max _{\sigma \in \mathrm{F}} \max _{\widetilde{N} \leq \mathbb{N}} \sum_{x} \operatorname{Tr} \tilde{\mathbb{N}}_{x} \Psi_{x}(\sigma)\right], \\
& \leqslant\left[1+\mathrm{R}_{\mathrm{F}}(\rho)\right]\left[1+\mathrm{R}_{\mathbb{F}}(\mathbb{M})\right] \max _{\sigma \in \mathrm{F}} \max _{\tilde{\mathbb{N}} \in \mathbb{F}} \max _{\widetilde{N} \leq \mathbb{N}} \sum_{x} \operatorname{Tr}\left[\tilde{N}_{x} \Psi_{x}(\sigma)\right], \\
& =\left[1+\mathrm{R}_{\mathrm{F}}(\rho)\right]\left[1+\mathrm{R}_{\mathbb{F}}(\mathbb{M})\right] \max _{\sigma \in \mathrm{F}} \max _{\mathbb{N} \in \mathbb{F}} P_{\text {succ }}^{\mathrm{D}}(\Psi, \sigma, \tilde{\mathbb{N}}) .
\end{aligned}
$$

In the first inequality, we use the definition of the generalized robustness from which we get $\rho \leqslant\left[1+\mathrm{R}_{\mathrm{F}}(\rho)\right] \sigma^{*}$ and since $\Psi_{x}$ are linear maps we have $\Psi_{x}(\rho) \leqslant\left[1+\mathrm{R}_{\mathrm{F}}(\rho)\right] \Psi_{x}\left(\sigma^{*}\right), \forall x$. In the second inequality we allow ourselves to maximize over all free states. In the third inequality, we use the definition of the generalized robustness from which we get $M_{a} \leqslant[1+$ $\left.\mathrm{R}_{\mathbb{M}}(\mathbb{M})\right] \tilde{N}_{a}^{*}, \forall a$. In the fourth inequality, we allow ourselves to maximize over all free measurements.

Proof. The proof for the lower bound for multiobject subchannel exclusion follows similar arguments.

\section{Achieving upper bound for discrimination and lower bound for exclusion}

Lemma 1. (Dual SDPs for generalized robustness) The generalized robustness of resource of a state $\rho$ and a measurement $\mathbb{M}=\left\{M_{x}\right\}, x \in\{1, \ldots, k\}$ can be written as

$$
\begin{gathered}
\mathrm{R}_{\mathrm{F}}(\rho)=\max _{Z} \operatorname{Tr}[(Z) \rho]-1, \\
\text { s.t. } Z \geqslant 0,
\end{gathered}
$$

$$
\begin{gathered}
\operatorname{Tr}[Z \sigma] \leqslant 1, \quad \forall \sigma \in \mathrm{F}, \\
\mathrm{R}_{\mathbb{F}}(\mathbb{M})=\max _{\left\{Z_{x}\right\}} \sum_{x} \operatorname{Tr}\left[Z_{x} M_{x}\right]-1, \\
\text { s.t. } Z_{x} \geqslant 0, \quad \forall x, \\
\sum_{x} \operatorname{Tr}\left[Z_{x} N_{x}\right] \leqslant 1, \quad \forall \mathbb{N} \in \mathbb{F} .
\end{gathered}
$$

These are the dual SDP formulations of the generalized robustnesses of resource for states and measurements.

Proof. (of result 2A) Given any state-measurement pair $(\rho, \mathbb{M})$, we want to find a suitable subchannel game $\Psi$ so that we achieve the upper bound in Eq. (B3). We start by noting that lemma 1 is a refined version of the hyperplane separation theorem, from which we can extract positive semidefinite operators $Z^{\rho},\left\{Z_{x}^{\mathbb{M}}\right\}, x \in\{1, \ldots, k\}$ satisfying the conditions (A1) and (A2). Therefore the construction of the set of subchannels from the previous section applies here as well. We 
then continue from (A8) which can now be rewritten as

$$
\begin{aligned}
P_{\text {succ }}^{\mathrm{D}}\left(\Psi^{(\rho, \mathbb{M}, n)}, \rho, \mathbb{M}\right) & \geqslant \alpha \operatorname{Tr}\left[Z^{\rho} \rho\right] \sum_{y=1}^{k} \operatorname{Tr}\left[M_{y} Z_{y}^{\mathbb{M}}\right], \\
& =\alpha\left[1+\mathrm{R}_{\mathrm{F}}(\rho)\right]\left[1+\mathrm{R}_{\mathbb{F}}(\mathbb{M})\right] .
\end{aligned}
$$

The equality follows from (B4a) and (B5a). We now analyze the fully free player. Similarly, we now choose the subchannel game given by $\Psi^{(\rho, \mathbb{M}, n \rightarrow \infty)}$ and invoking (A11) we have

$$
\max _{\sigma \in \mathrm{F}} \max _{\mathbb{N} \in \mathbb{F}} P_{\text {succ }}^{\mathrm{D}}\left(\Psi^{(\rho, \mathbb{M}, n \rightarrow \infty)}, \sigma, \mathbb{N}\right) \leqslant \alpha .
$$

We now analyze the ratio of interest with this particular subchannel game and have

$$
\begin{aligned}
& \frac{P_{\text {succ }}^{\mathrm{D}}\left(\Psi^{(\rho, \mathbb{M}, n \rightarrow \infty)}, \rho, \mathbb{M}\right)}{\max _{\sigma \in \mathrm{F}} \max _{\mathbb{N} \in \mathbb{F}} P_{\text {succ }}^{\mathrm{D}}\left(\Psi^{(\rho, \mathbb{M}, n \rightarrow \infty)}, \sigma, \mathbb{N}\right)} \\
& \geqslant \frac{\alpha\left[1+\mathrm{R}_{\mathrm{F}}(\rho)\right]\left[1+\mathrm{R}_{\mathbb{F}}(\mathbb{M})\right]}{\max _{\sigma \in \mathrm{F}} \max _{\mathbb{N} \in \mathbb{F}} P_{\text {succ }}^{\mathrm{D}}\left(\Psi^{(\rho, \mathbb{M}, n \rightarrow \infty)}, \sigma, \mathbb{N}\right)} \\
& \geqslant \frac{\alpha\left[1+\mathrm{R}_{\mathrm{F}}(\rho)\right]\left[1+\mathrm{R}_{\mathbb{F}}(\mathbb{M})\right]}{\alpha} \\
& =\left[1+\mathbf{R}_{\mathrm{F}}(\rho)\right]\left[1+\mathbf{R}_{\mathbb{F}}(\mathbb{M})\right] .
\end{aligned}
$$

In the first inequality, we used (B6) while in the second, we used (B7). Putting together (B8) and (B3), we obtain

$$
\begin{gathered}
\left.\frac{P_{\text {succ }}^{\mathrm{D}}\left(\Psi^{(\rho, \mathbb{M}, n \rightarrow \infty)}, \rho, \mathbb{M}\right)}{\max _{\substack{\sigma \in \mathrm{F} \\
\mathbb{N} \in \mathbb{F}}} P_{\text {succ }}^{\mathrm{D}}(\Psi(\rho, \mathbb{M}, n \rightarrow \infty)}, \sigma, \mathbb{N}\right) \\
=\left[1+\mathrm{R}_{\mathrm{F}}(\rho)\right]\left[1+\mathrm{R}_{\mathbb{F}}(\mathbb{M})\right] .
\end{gathered}
$$

as desired.

Lemma 2. (Dual SDPs for weight) The weight of resource of a state $\rho$ and a measurement $\mathbb{M}=\left\{M_{x}\right\}, x \in\{1, \ldots, k\}$ can be written as

$$
\begin{gathered}
\mathrm{W}_{\mathrm{F}}(\rho)=\max _{Y} \operatorname{Tr}[(-Y) \rho]+1, \\
\text { s.t. } Y \geqslant 0, \\
\operatorname{Tr}[Y \sigma] \geqslant 1, \quad \forall \sigma \in \mathrm{F}, \\
\mathrm{W}_{\mathbb{F}}(\mathbb{M})=\max _{\left\{Y_{x}\right\}} \sum_{x} \operatorname{Tr}\left[\left(-Y_{x}\right) M_{x}\right]+1, \\
\text { s.t. } Y_{x} \geqslant 0, \quad \forall x, \\
\sum_{x} \operatorname{Tr}\left[Y_{x} N_{x}\right] \geqslant 1, \quad \forall \mathbb{N} \in \mathbb{F} .
\end{gathered}
$$

These are the dual SDP formulations of the weights of resource for states and measurements.

Proof. (of result 2B) This proof follows a similar logic to that of the robustness, and we write down for completeness. Given any state-measurement pair $(\rho, \mathbb{M})$, we want to find a suitable subchannel game $\Psi$ so that we achieve the lower bound in Eq. (B2). The construction of the set of subchannels form the previous section applies here as well. We then continue from (A17) which can now be rewritten as

$$
\begin{aligned}
P_{\mathrm{err}}^{\mathrm{E}}\left(\Psi^{(\rho, \mathbb{M})} \rho, \mathbb{M}\right) & \leqslant \beta \operatorname{Tr}\left[Y^{\rho} \rho\right] \sum_{y=1}^{k} \operatorname{Tr}\left[M_{y} Y_{y}^{\mathbb{M}}\right], \\
& =\beta\left[1-\mathrm{W}_{\mathrm{F}}(\rho)\right]\left[1-\mathrm{W}_{\mathbb{F}}(\mathbb{M})\right] .
\end{aligned}
$$

The equality follows from (B9a) and (B10a). We now analyze the fully free player and invoke (A21), which reads

$$
\min _{\sigma \in \mathrm{F}} \min _{\mathbb{N} \in \mathbb{F}} P_{\mathrm{err}}^{\mathrm{E}}\left(\Psi^{(\rho, \mathbb{M})}, \sigma, \mathbb{N}\right) \geqslant \beta .
$$

We now analyze the ratio of interest with this particular subchannel game and have

$$
\begin{aligned}
& \frac{P_{\mathrm{err}}^{\mathrm{E}}\left(\Psi^{(\rho, \mathbb{M})}, \rho, \mathbb{M}\right)}{\min _{\sigma \in \mathrm{F}} \min _{\mathbb{N} \in \mathbb{F}} P_{\mathrm{err}}^{\mathrm{E}}\left(\Psi^{(\rho, \mathbb{M})}, \sigma, \mathbb{N}\right)} \\
& \leqslant \frac{\beta\left[1-\mathrm{W}_{\mathrm{F}}(\rho)\right]\left[1-\mathrm{W}_{\mathbb{F}}(\mathbb{M})\right]}{\min _{\sigma \in \mathrm{F}} \min _{\mathbb{N} \in \mathbb{F}} P_{\mathrm{err}}^{\mathrm{E}}\left(\Psi^{(\rho, \mathbb{M})}, \sigma, \mathbb{N}\right)} \\
& \leqslant \frac{\beta\left[1-\mathrm{W}_{\mathrm{F}}(\rho)\right]\left[1-\mathrm{W}_{\mathbb{F}}(\mathbb{M})\right]}{\beta} \\
& =\left[1-\mathrm{W}_{\mathrm{F}}(\rho)\right]\left[1-\mathrm{W}_{\mathbb{F}}(\mathbb{M})\right] \text {. }
\end{aligned}
$$

In the first inequality, we used (B11), while in the second, we used (B12). Putting together (B13) and the lower bound in Eq. (B2), we obtain

$$
\frac{P_{\mathrm{err}}^{\mathrm{E}}\left(\Psi^{(\rho, \mathbb{M})}, \rho, \mathbb{M}\right)}{\min _{\substack{\sigma \in \mathrm{F} \\ \mathbb{N} \in \mathbb{F}}} P_{\mathrm{err}}^{\mathrm{E}}\left(\Psi^{(\rho, \mathbb{M})}, \sigma, \mathbb{N}\right)}=\left[1-\mathrm{W}_{\mathrm{F}}(\rho)\right]\left[1-\mathrm{W}_{\mathbb{F}}(\mathbb{M})\right],
$$

as desired.

\section{APPENDIX C: PROOF OF RESULT 3}

Result $3 A$. The maximum gap between the order plusinfinity mutual information of any state-measurement pair $(\rho, \mathbb{M})$ when compared to the best fully free statemeasurement pair is upper bounded as

$$
\begin{aligned}
& \max _{\Lambda}\left\{I_{+\infty}\left(X_{\Lambda, \rho} ; G_{\mathbb{M}}\right)-\max _{\sigma \in \mathrm{F}} \max _{\mathbb{N} \in \mathbb{F}} I_{+\infty}\left(X_{\Lambda, \sigma} ; G_{\mathbb{N}}\right)\right\} \\
& \leqslant \log _{2}\left[1+\mathrm{R}_{\mathrm{F}}(\rho)\right]+\log _{2}\left[1+\mathrm{R}_{\mathbb{F}}(\mathbb{M})\right],
\end{aligned}
$$

with the maximization over all ensembles of channels.

Proof. The plus-infinity mutual information between classical random variables $X_{\Lambda, \rho}$ and $G_{\mathbb{M}}$ is given by [59]

$$
I_{+\infty}\left(X_{\Lambda, \rho} ; G_{\mathbb{M}}\right)=+\left[H_{+\infty}\left(X_{\Lambda, \rho}\right)-H_{+\infty}\left(X_{\Lambda, \rho} \mid G_{\mathbb{M}}\right)\right],
$$

with $H_{+\infty}\left(X_{\Lambda, \rho}\right)=-\log _{2}\left(\max _{x} p(x)\right), \quad H_{+\infty}\left(X_{\Lambda, \rho} \mid G_{\mathbb{M}}\right)=$ $-\log _{2}\left(\sum_{g} \max _{x} p(g, x)\right) \quad$ with $p(g, x)=p(g \mid x) p(x)$. We have $\quad p(g \mid x)=\operatorname{Tr}\left(M_{g} \Lambda_{x}(\rho)\right) \quad$ and $\quad H_{+\infty}\left(X_{\Lambda, \rho} \mid G_{\mathbb{M}}\right)=$ $-\log _{2} \sum_{g} \max _{x} \operatorname{Tr}\left[M_{g} \Lambda_{x}(\rho)\right] p(x)$. Considering $f_{g}(x)=$ $\operatorname{Tr}\left[M_{g} \Lambda_{x}(\rho)\right] p(x)$ and using

$$
\max _{x} f_{g}(x)=\max _{\{p(x \mid g)\}} \sum_{x} p(x \mid g) f_{g}(x)
$$


we have

$$
\begin{aligned}
& H_{+\infty}\left(X_{\Lambda, \rho} \mid G_{\mathbb{M}}\right) \\
& \quad=-\log _{2} \sum_{g} \max _{\{p(x \mid g)\}} \sum_{x} p(x \mid g) f_{g}(x), \\
& \quad=-\log _{2} \sum_{g} \max _{\{p(x \mid g)\}} \sum_{x} p(x \mid g) \operatorname{Tr}\left[M_{g} \Lambda_{x}(\rho)\right] p(x), \\
& =-\log _{2} \max _{\{p(x \mid g)\}} \sum_{x} \operatorname{Tr}\left[\left(\sum_{g} p(x \mid g) M_{g}\right) \Lambda_{x}(\rho)\right] p(x), \\
& =-\log _{2} \max _{\mathbb{N}<\mathbb{M}} \sum_{x} \operatorname{Tr}\left[N_{x} \Lambda_{x}(\rho)\right] p(x), \\
& =-\log _{2} P_{\text {succ }}^{\mathrm{D}}(\Lambda, \mathbb{M}, \rho) .
\end{aligned}
$$

We then have the following expression:

$$
\begin{aligned}
I_{+\infty} & \left(X_{\Lambda, \rho} ; G_{\mathbb{M}}\right)-\max _{\sigma \in \mathrm{F}} \max _{\mathbb{N} \in \mathbb{F}} I_{+\infty}\left(X_{\Lambda, \sigma} ; G_{\mathbb{N}}\right) \\
& =-H_{+\infty}\left(X_{\Lambda, \rho} \mid G_{\mathbb{M}}\right)-\max _{\sigma \in \mathrm{F}} \max _{\mathbb{N} \in \mathbb{F}}-H_{+\infty}\left(X_{\Lambda, \sigma} \mid G_{\mathbb{N}}\right), \\
& =\log _{2}\left[P_{\text {succ }}^{\mathrm{D}}(\Lambda, \mathbb{M}, \rho)\right]-\max _{\sigma \in \mathrm{F}} \max _{\mathbb{N} \in \mathbb{F}} \log _{2}\left[P_{\text {succ }}^{\mathrm{D}}(\Lambda, \mathbb{N}, \sigma)\right], \\
& =\log _{2}\left\{\frac{P_{\text {succ }}^{\mathrm{D}}(\Lambda, \mathbb{M}, \rho)}{\max _{\mathbb{N} \in \mathbb{F}} \max _{\sigma \in \mathrm{F}} P_{\text {succ }}^{\mathrm{D}}(\Lambda, \mathbb{N}, \sigma)}\right\} .
\end{aligned}
$$

We now maximize over all ensembles of channels and using result $2 \mathrm{~A}$, we obtain the claim in Eq. (C1).

Result $3 B$. The maximum gap between the order minusinfinity mutual information of any state-measurement pair $(\rho, \mathbb{M})$ when compared to the best fully free statemeasurement pair is upper bounded as

$$
\begin{gathered}
\max _{\Lambda}\left\{I_{-\infty}\left(X_{\Lambda, \rho} ; G_{\mathbb{M}}\right)-\max _{\sigma \in \mathrm{F}} \max _{\mathbb{N} \in \mathbb{F}} I_{-\infty}\left(X_{\Lambda, \sigma} ; G_{\mathbb{N}}\right)\right\} \\
\leqslant-\log _{2}\left[1-\mathrm{W}_{\mathrm{F}}(\rho)\right]-\log _{2}\left[1-\mathrm{W}_{\mathbb{F}}(\mathbb{M})\right],
\end{gathered}
$$

with the maximization over all ensembles of channels.
Proof. The minus-infinity mutual information between classical random variables $X_{\Lambda, \rho}$ and $G_{\mathbb{M}}$ is given by [50]

$$
I_{-\infty}\left(X_{\Lambda, \rho} ; G_{\mathbb{M}}\right)=-\left[H_{-\infty}\left(X_{\Lambda, \rho} \mid G_{\mathbb{M}}\right)-H_{-\infty}\left(X_{\Psi}\right)\right],
$$

with $H_{-\infty}\left(X_{\Lambda, \rho}\right)=-\log _{2}\left(\min _{x} p(x)\right), \quad H_{-\infty}\left(X_{\Lambda, \rho} \mid G_{\mathbb{M}}\right)=$ $-\log _{2} \sum_{g} \min _{x} p(g, x), \quad p(g, x)=p(g \mid x) p(x) . \quad$ Using $p(g \mid x)=\operatorname{Tr}\left[M_{g} \Lambda_{x}(\rho)\right]$ then $H_{-\infty}\left(X_{\Lambda, \rho} \mid G_{\mathbb{M}}\right)=$ $-\log _{2} \sum_{g} \min _{x} \operatorname{Tr}\left[M_{g} \Lambda_{x}(\rho)\right] p(x)$. Considering $f_{g}(x)=$ $\operatorname{Tr}\left[M_{g} \Lambda_{x}(\rho)\right] p(x)$ and using

$$
\min _{x} f_{g}(x)=\min _{\{p(x \mid g)\}} \sum_{x} p(x \mid g) f_{g}(x),
$$

we have

$$
\begin{aligned}
& H_{-\infty}\left(X_{\Lambda, \rho} \mid G_{\mathbb{M}}\right) \\
& \quad=-\log _{2} \sum_{g} \min _{\{p(x \mid g)\}} \sum_{x} p(x \mid g) f_{g}(x), \\
& \quad=-\log _{2} \sum_{g} \min _{\{p(x \mid g)\}} \sum_{x} p(x \mid g) \operatorname{Tr}\left[M_{g} \Lambda_{x}(\rho)\right] p(x), \\
& \quad=-\log _{2} \min _{\{p(x \mid g)\}} \sum_{x} \operatorname{Tr}\left[\left(\sum_{g} p(x \mid g) M_{g}\right) \Lambda_{x}(\rho)\right] p(x), \\
& =-\log _{2} \min _{\mathbb{N}<\mathbb{M}} \sum_{x} \operatorname{Tr}\left[N_{x} \Lambda_{x}(\rho)\right] p(x), \\
& \quad=-\log _{2} P_{\mathrm{err}}^{\mathrm{E}}(\Lambda, \mathbb{M}, \rho) .
\end{aligned}
$$

We then have the following expression:

$$
\begin{aligned}
& I_{-\infty}\left(X_{\Lambda, \rho} ; G_{\mathbb{M}}\right)-\max _{\sigma \in \mathrm{F}} \max _{\mathbb{N} \in \mathbb{F}} I_{-\infty}\left(X_{\Lambda, \rho} ; G_{\mathbb{N}}\right) \\
& =H_{-\infty}\left(X_{\Lambda, \rho} \mid G_{\mathbb{M}}\right)-\max _{\sigma \in \mathrm{F}} \max _{\mathbb{N} \in \mathbb{F}} H_{-\infty}\left(X_{\Lambda, \sigma} \mid G_{\mathbb{N}}\right), \\
& =-\log _{2}\left[P_{\mathrm{err}}^{\mathrm{E}}(\Lambda, \mathbb{M}, \rho)\right]-\max _{\sigma \in \mathrm{F}} \max _{\mathbb{N} \in \mathbb{F}}-\log _{2}\left[P_{\mathrm{err}}^{\mathrm{E}}(\Lambda, \mathbb{N}, \sigma)\right], \\
& =-\log _{2}\left[P_{\mathrm{err}}^{\mathrm{E}}(\Lambda, \mathbb{M}, \rho)\right]+\min _{\sigma \in \mathrm{F}} \min _{\mathbb{N} \in \mathbb{F}} \log _{2}\left[P_{\mathrm{err}}^{\mathrm{E}}(\Lambda, \mathbb{N}, \sigma)\right], \\
& =-\left\{\log _{2}\left[P_{\mathrm{err}}^{\mathrm{E}}(\Lambda, \mathbb{M}, \rho)\right]-\min _{\sigma \in \mathrm{F}} \min _{\mathbb{N} \in \mathbb{F}} \log _{2}\left[P_{\mathrm{err}}^{\mathrm{E}}(\Lambda, \mathbb{N}, \sigma)\right]\right\}, \\
& =-\log _{2}\left\{\frac{P_{\mathrm{err}}^{\mathrm{Q}}(\Psi, \mathbb{M}, \rho)}{\min _{\sigma \in \mathrm{F}} \min _{\mathbb{N} \in \mathbb{F}} P_{\mathrm{err}}^{\mathrm{E}}(\Psi, \mathbb{N}, \sigma),}\right\} .
\end{aligned}
$$

We now maximize over all ensembles of channels and using result 2B we obtain the claim in Eq. (C4).
[1] M. Horodecki and J. Oppenheim, Int. J. Mod. Phys. B 27, 1345019 (2012).

[2] E. Chitambar and G. Gour, Rev. Mod. Phys. 91, 025001 (2019).

[3] C. H. Bennett, G. Brassard, S. Popescu, B. Schumacher, J. A. Smolin, and W. K. Wootters, Phys. Rev. Lett. 76, 722 (1996).

[4] C. H. Bennett, H. J. Bernstein, S. Popescu, and B. Schumacher, Phys. Rev. A 53, 2046 (1996).

[5] T. Baumgratz, M. Cramer, and M. B. Plenio, Phys. Rev. Lett. 113, 140401 (2014).

[6] A. Winter and D. Yang, Phys. Rev. Lett. 116, 120404 (2016).

[7] J. Åberg, Phys. Rev. Lett. 113, 150402 (2014).

[8] G. Gour and R. W. Spekkens, New J. Phys. 10, 033023 (2008).
[9] I. Marvian and R. W. Spekkens, New J. Phys. 15, 033001 (2013).

[10] J. Aberg, arXiv:quant-ph/0612146 [quant-ph].

[11] M. Horodecki, P. Horodecki, and J. Oppenheim, Phys. Rev. A 67, 062104 (2003).

[12] D. Janzing, P. Wocjan, R. Zeier, R. Geiss, and T. Beth, Int. J. Theor. Phys. 39, 2717 (2000).

[13] F. G. S. L. Brandão, M. Horodecki, J. Oppenheim, J. M. Renes, and R. W. Spekkens, Phys. Rev. Lett. 111, 250404 (2013).

[14] M. Horodecki and J. Oppenheim, Nat. Commun. 4, 2059 (2013). 
[15] T. Theurer, N. Killoran, D. Egloff, and M. B. Plenio, Phys. Rev. Lett. 119, 230401 (2017).

[16] M. Howard and E. Campbell, Phys. Rev. Lett. 118, 090501 (2017).

[17] R. Takagi and Q. Zhuang, Phys. Rev. A 97, 062337 (2018).

[18] S. Bhattacharya, B. Bhattacharya, and A. S. Majumdar, arXiv: 1803.06881.

[19] E. Wakakuwa, arXiv:1709.07248.

[20] N. Anand and T. A. Brun, arXiv:1903.03880.

[21] M. Horodecki, J. Oppenheim, and R. Horodecki, Phys. Rev. Lett. 89, 240403 (2002).

[22] M. Oszmaniec, L. Guerini, P. Wittek, and A. Acín, Phys. Rev. Lett. 119, 190501 (2017).

[23] L. Guerini, J. Bavaresco, M. T. Cunha, and A. Acín, J. Math. Phys. 58, 092102 (2017).

[24] P. Skrzypczyk and N. Linden, Phys. Rev. Lett. 122, 140403 (2019).

[25] M. Oszmaniec and T. Biswas, Quantum 3, 133 (2019).

[26] J. Barrett, N. Linden, S. Massar, S. Pironio, S. Popescu, and D. Roberts, Phys. Rev. A 71, 022101 (2005).

[27] J. I. de Vicente, J. Phys. A: Math. Theor. 47, 424017 (2014).

[28] R. Gallego and L. Aolita, Phys. Rev. X 5, 041008 (2015).

[29] I. Devetak, A. W. Harrow, and A. J. Winter, IEEE Trans. Inf. 54, 4587 (2008).

[30] Z.-W. Liu and A. Winter, arXiv:1904.04201.

[31] Y. Liu and X. Yuan, Phys. Rev. Res. 2, 012035 (2020).

[32] T. Theurer, D. Egloff, L. Zhang, and M. B. Plenio, Phys. Rev. Lett. 122, 190405 (2019).

[33] S. Pirandola, R. Laurenza, C. Ottaviani, and L. Banchi, Nat. Commun. 8, 15043 (2017).

[34] G. Vidal and R. Tarrach, Phys. Rev. A 59, 141 (1999).

[35] D. Cavalcanti and P. Skrzypczyk, Phys. Rev. A 93, 052112 (2016).

[36] M. Piani and J. Watrous, Phys. Rev. Lett. 114, 060404 (2015).

[37] M. Piani, M. Cianciaruso, T. R. Bromley, C. Napoli, N. Johnston, and G. Adesso, Phys. Rev. A 93, 042107 (2016).

[38] C. Napoli, T. R. Bromley, M. Cianciaruso, M. Piani, N. Johnston, and G. Adesso, Phys. Rev. Lett. 116, 150502 (2016).
[39] I. Šupić, P. Skrzypczyk, and D. Cavalcanti, Phys. Rev. A 99, 032334 (2019).

[40] P. Lipka-Bartosik and P. Skrzypczyk, Phys. Rev. Res. 2, 023029 (2020).

[41] R. Uola, T. Kraft, J. Shang, X.-D. Yu, and O. Gühne, Phys. Rev. Lett. 122, 130404 (2019).

[42] C. Carmeli, T. Heinosaari, T. Miyadera, and A. Toigo, J. Math. Phys. 60, 122202 (2019).

[43] M. Lewenstein and A. Sanpera, Phys. Rev. Lett. 80, 2261 (1998).

[44] A. C. Elitzur, S. Popescu, and D. Rohrlich, Phys. Lett. A 162, 25 (1992).

[45] P. Skrzypczyk, M. Navascués, and D. Cavalcanti, Phys. Rev. Lett. 112, 180404 (2014).

[46] K. Bu, N. Anand, and U. Singh, Phys. Rev. A 97, 032342 (2018).

[47] P. Skrzypczyk, I. Šupić, and D. Cavalcanti, Phys. Rev. Lett. 122 130403 (2019).

[48] J. Mori, Phys. Rev. A 101, 032331 (2020).

[49] R. Takagi and B. Regula, Phys. Rev. X 9, 031053 (2019).

[50] A. F. Ducuara and P. Skrzypczyk, arXiv:1909.10486 [Phys. Rev. Lett. (to be published)].

[51] R. Uola, T. Bullock, T. Kraft, J.-P. Pellonpää, and N. Brunner, arXiv:1909.10484 [Phys. Rev. Lett. (to be published)].

[52] C. Sparaciari, L. del Rio, C. M. Scandolo, P. Faist, and J. Oppenheim, Quantum 4, 259 (2020).

[53] Y. Guryanova, S. Popescu, A. J. Short, R. Silva, and P. Skrzypczyk, Nat. Commun. 7, 12049 (2016).

[54] N. Yunger Halpern, P. Faist, J. Oppenheim, and A. Winter, Nat. Commun. 7, 12051 (2016).

[55] M. Lostaglio, D. Jennings, and T. Rudolph, New J. Phys. 19 , 043008 (2017).

[56] R. T. Rockafellar, Convex Analysis (Princeton University Press, Princeton, NJ, 2015).

[57] R. Takagi, B. Regula, K. Bu, Z.-W. Liu, and G. Adesso, Phys. Rev. Lett. 122, 140402 (2019).

[58] M. Wilde, Quantum Information Theory (Cambridge University Press, Cambridge, 2017).

[59] R. Renner, arXiv:quant-ph/0512258. 